Research Paper

\title{
Choline Protects Against Cardiac Hypertrophy Induced by Increased After-load
}

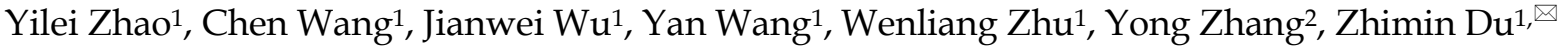 \\ 1. Institute of Clinical Pharmacology, the Second Affiliated Hospital of Harbin Medical University, China; \\ 2. Department of Pharmacology, the State-Province Key Laboratories of Biomedicine- Pharmaceutics of China, Key Laboratory of Cardi- \\ ovascular Research, Harbin Medical University, China.
}

$\square$ Corresponding author: Prof. Zhimin Du, Institute of Clinical Pharmacology, the Second Affiliated Hospital of Harbin Medical University, Harbin, 150086, China. Tel: 86451 86605353; Fax: 86451 86605353; E-mail: dzm1956@126.com.

(C) Ivyspring International Publisher. This is an open-access article distributed under the terms of the Creative Commons License (http://creativecommons.org/ licenses/by-nc-nd/3.0/). Reproduction is permitted for personal, noncommercial use, provided that the article is in whole, unmodified, and properly cited.

Received: 2013.01.29; Accepted: 2013.02.26; Published: 2013.03.08

\begin{abstract}
Background: Although inadequate intake of essential nutrient choline has been known to significantly increase cardiovascular risk, whether additional supplement of choline offering a protection against cardiac hypertrophy remain unstudied.

Methods: The effects of choline supplements on pathological cardiac hypertrophic growth induced by transverse aorta constriction (TAC) for three weeks and cardiomyocyte hypertrophy in cultured cells induced by isoproterenol (ISO) $10 \mu \mathrm{M}$ for $48 \mathrm{~h}$ stimulation were investigated. Western blot analysis and real-time PCR were used to determine the expression of ANP, BNP, $\beta-M H C$, miR-I33a and Calcineurin.

Results: Administration of $14 \mathrm{mg} / \mathrm{kg}$ choline to mice undergone TAC effectively attenuated the cardiac hypertrophic responses, as indicated by the reduced heart weight, left ventricular weight, ventricular thickness, and reduced expression of biomarker genes of cardiac hypertrophy. This anti-hypertrophic efficacy was reproduced in a cellular model of cardiomyocyte hypertrophy induced by isoproterenol in cultured neonatal cardiomyocytes. Our results further showed that choline rescued the aberrant downregulation of the muscle-specific microRNA miR-133a expression, a recently identified anti-hypertrophic factor, and restored the elevated calcineurin protein level, the key signaling molecule for the development of cardiac hypertrophy. These effects of choline were abolished by the $M_{3} m A C h R$-specific antagonist 4-DAMP.

Conclusion: Our study unraveled for the first time the cardioprotection of choline against cardiac hypertrophy, with correction of expression of miR-133a and calcineurin as a possible mechanism. Our findings suggest that choline supplement may be considered for adjunct anti-hypertrophy therapy.
\end{abstract}

Key words: choline; cardiac hypertrophy; cardiomyocyte; miR-133a; Calcineurin.

\section{Introduction}

Choline is classified as nutrient; it plays an extremely wide range of biological roles in human health and disease [1]. Inadequate intake or abnormal metabolism of choline is associated with the increased risk of cardiovascular disease and cancer [2,3]. Our previous study investigated cardioprotection of choline in preventing ischemic heart disease, the most common cause of lethal heart failure [4]. Activation of $\mathrm{M}_{3}$ muscarinic acetylcholine receptor (mAChR) pathway by choline provided multiple protective effects on the injured heart via restoration of the normal intracellular free $\mathrm{Ca}^{2+}$ concentration $[5,6]$, reduction of intracellular reactive oxygen species (ROS) [7], and especially alteration of miRNA expression profile of 
cardiac myocytes [4], implying the central role of choline-induced cardioprotection for myocardial ischemia.

Compared with myocardial ischemia, cardiac hypertrophy represents another potent risk for cardiovascular diseases such as cardiac arrhythmias and heart failure, and even sudden death [8]. In response to an increase in pressure load due to insufficient blood supply, the heart must work harder than that under normal condition. Along with the functional change, it is accompanied by significant enlargement of heart mass, increase in protein synthesis, reorganization of sarcomere, activation of the fetal gene expression, and increase in cardiac fibrosis [9]. Therefore, it is very important to cure or reverse transverse aorta constriction (TAC)-induced hypertrophy before its transition to heart failure.

In the present study, we investigated whether choline supplements could be used as prevention approach against TAC-induced hypertrophy. The muscle-specific microRNA miR-133a has been shown to critically determine the development of cardiac hypertrophy and its downregulation is a hallmark of this cardiac condition $[10,11]$. As choline fulfills its ischemic cardioprotection mainly as an agonist of $\mathrm{M}_{3}$ $\mathrm{mAChR}[5,12,13]$, the role of choline/ $\mathrm{M}_{3} \mathrm{mAChR}$ pathway in cardiac hypertrophy induced by TAC was explored here. Furthermore, network analysis was performed to elucidate the potential signaling pathways that choline might be involved in. Our findings support that supplemental choline affords sound benefits to TAC-induced hypertrophy.

\section{Materials and Methods}

\section{Ethics statement}

All procedures for the use of animals were pre-approved by the Experimental Animal Ethic Committee of Harbin Medical University, China (Animal Experimental Ethical Inspection Protocol No. 2009104). Use of animals was complied with the Guide for the Care and Use of Laboratory Animals published by the US National Institutes of Health (NIH Publication No.85-23, revised 1996).

\section{Cardiac hypertrophy model of mice}

Healthy male adult Kunming mice were obtained from the Experimental Animal Center of Harbin Medical University. Mice received standard diet and water, and were kept under standard conditions in the vivarium. Adult mice $(24 \mathrm{~g} \sim 28 \mathrm{~g})$ were anesthetized with pentobarbital sodium $(60 \mathrm{mg} / \mathrm{kg}$, i.p.) and randomly divided into four experimental groups: Sham, transverse aorta constriction (TAC), TAC+Choline (14 mg/kg, i.p.), TAC+Choline+4DAMP $(14 \mathrm{mg} / \mathrm{kg}$ and $0.7 \mu \mathrm{g} / \mathrm{kg}$, respectively, i.p.)
$[5,7,14]$. The mouse model of cardiac hypertrophy was established by TAC as described before [15]. After surgery, animals were placed in the warm environment with a breathing machine (UGO BASILE, Biological Research Apparatus, Italy) at the respiratory rate of 95 breaths/min with a tidal volume of $0.5 \mathrm{ml}$ until restoration of spontaneous breathing. The sham-operated mice underwent the same surgical procedures without aorta banding. Three days after $\mathrm{TAC}$, the mice were injected with choline (Sigma, St Louis, MO, USA) or choline+4-DAMP combination daily. For co-administration of choline and 4-DAMP (Sigma, St Louis, MO, USA), 4-DAMP was injected 30 min before choline treatment. After 3 weeks, the survived animals were sacrificed, and hearts were quickly isolated and weighed in cold buffer. The left ventricle was rapidly frozen in liquid nitrogen and stored in $-80^{\circ} \mathrm{C}$ freezer for subsequent western blot and RT-PCR experiments.

\section{Echocardiography measurement and histo- logical analysis}

Three weeks after TAC operation, mice were again anesthetized with pentobarbital sodium (60 $\mathrm{mg} / \mathrm{kg}$ i.p.), placed on the experimental platform. Transthoracic echocardiography was performed using an in vivo ultrasound imaging system with a $40-\mathrm{MHz}$ transducer (Cold Spring Biotech Corp, Taiwan). Two-dimensional images and M-mode recordings of left ventricular area were obtained from the parasternal long-axis view. Heart rate and ventricular wall thickness were measured and the percentage of ejection fraction (EF) was calculated.

For histological analysis, the hearts were fixed with $4 \%$ paraformaldehyde ( $\mathrm{pH} 7.4$ ) for $48 \mathrm{~h}$. Tissues were embedded in paraffin, sectioned into 5- $\mu \mathrm{m}$ slices, and stained with standard hematoxylin and eosin (HE). To calculate the cell surface area, fifty cell cross-sections from three hearts were measured using the Image-Pro plus Data Analysis Software (Version 5.0.1, Media Cybernetics, Silver Spring, MD, USA). The average value was used for analysis.

\section{Primary culture of neonatal rat cardiomyo- cytes}

Neonatal rat ventricular myocytes (NRVMs) were isolated from one day old Sprague-Dawley rats. Briefly, the hearts were quickly collected and digested with collagenase II. The preparation was centrifuged (2000 rpm, $180 \mathrm{~s}$ ) and redissolved in DMEM. Two hours later, NRVMs were collected, and incubated in a humidified incubator in the presence of $95 \% \mathrm{O}_{2}$ and $5 \% \mathrm{CO}_{2}$.

NRVMs were exposed to isoproterenol (ISO, 10 $\mu \mathrm{M}$, Sigma, St Louis, MO, USA) for $48 \mathrm{~h}$ in order to 
induce cardiomyocyte hypertrophy. NRVMs were pre-incubated with choline $(0.5 \mathrm{mM})[5,7,14]$ for 30 min before ISO treatment, and in some experiments, 4-DAMP $(3 \mathrm{nM})[5,7,14]$ was added $30 \mathrm{~min}$ before choline pretreatment. Then, NRVMs were incubated for another $48 \mathrm{~h}$.

\section{Immunocytochemistry}

Cultured NRVMs were washed three times with PBS and then fixed with $4 \%$ paraformaldehyde for 0.5 h, permeablized with $0.4 \%$ Triton X-100 for $1 \mathrm{~h}$, and finally blocked for $1 \mathrm{~h}$ in PBS with goat serum. Next, the cells were incubated with anti-a-actinin (Sigma, St Louis, MO, USA) at 1:200 dilution ratio overnight, followed by subsequent incubation with Alexa Fluor 594 antibody for $1 \mathrm{~h}$. The cell nuclei were counterstained with DAPI. Fluorescence images were analyzed with Image-Pro Plus Data Analysis Software (Version 5.0.1, Media Cybernetics, Silver Spring, MD, USA). Quantification of cell surface area by measuring random cells from three experiments, and the average value was used for analysis.

\section{Western blot analysis}

Western blot analysis was performed according to the procedures as previously described [13]. Following incubation with primary antibodies for $\beta$-MHC (Santa Cruz Biotechnology Inc.USA), calcineurin (Santa Cruz Biotechnology Inc. USA) and GAPDH (Kangcheng Shanghai. China) at $4^{\circ} \mathrm{C}$ overnight, the membranes were washed and incubated with secondary antibodies. Odyssey v1.2 software was used to quantify the western blot bands by measuring band intensity (area $\times \mathrm{OD}$ ) of each group.

\section{Quantitative reverse transcrip- tion-polymerase chain reaction}

Total RNA samples from left ventricular tissues and NRVMs were extracted with TRIZOL reagent (Invitrogen, USA). After the RNA concentration and purity had been qualified, $0.5 \mu \mathrm{g}$ RNA was used as a template for cDNA synthesis for reverse transcription reaction. Quantitative analysis of mRNA was executed with ABI 7500 fast Real Time PCR system (Applied Biosystems, USA). GAPDH and U6 were used as internal controls, respectively. Detailed sequences of essential primers are presented in Supplementary Material: Table S1.

\section{Statistics}

All data are expressed as mean \pm SEM. Statistical analysis was performed using one-way ANOVA followed by Bonferroni's test. Differences were considered as statistically significant when $p<0.05$.

\section{Results}

\section{Choline attenuates cardiac hypertrophy in vivo} and in vitro

Administration of choline to ischemic mice significantly attenuated TAC-induced hypertrophy, as identified by measurement of the ratio of heart weight over body weight (HW/BW) and that of left ventricular weight to BW (LVW/BW) (Fig. 1A). A similar result was found when comparing cross-sectional area of cardiomyocytes among different experimental groups (Fig. 1B and Supplementary Material: Fig. S1A). TAC-treated mice showed an obvious increase in the thickness of left ventricular posterior wall and interventricular septal due to compensatory hypertrophy. Echocardiography data also revealed that the thickness of left ventricular posterior wall and EF were significantly decreased by choline pretreatment. These effects were significantly weakened by 4-DAMP pretreatment, implying that activation of the $\mathrm{M}_{3} \mathrm{mAChR}$ signaling pathway was implicated in the actions of choline (Fig.1C and Supplementary Material: Fig. S1B-D). Furthermore, choline refrained the increased mRNA level of $\beta$-myosin heavy chain $(\beta-\mathrm{MHC})$, atrial natriuretic peptide (ANP), and brain natriuretic peptide (BNP) induced by TAC. The $\beta$-MHC protein level was also obviously down-regulated by choline in TAC mice. Addition of 4-DAMP significantly disturbed the beneficial effects of choline (Fig. 1D-1E and Supplementary Material: Fig. S1E). To see whether the anti-hypertrophic effect of choline could be reproduced in cultured myocardial cells, we established an in vitro model of hypertrophic NRVMs by ISO stimulation. Choline pretreatment markedly inhibited the increase of cell surface area induced by ISO (Fig. 2A-2B) and meanwhile decreased the ISO-elevated $\beta$-MHC mRNA and protein levels nearly to the control levels (Fig. 2C-2D). Taken together, these results confirmed the protective effects of choline against cardiac hypertrophy.

\section{Choline normalizes pathological expression of miR-133a}

The muscle-specific miR-133a was considered as hypertrophy predictor $[10,11]$, which was significantly down-regulated in cardiac hypertrophy. We found that choline was able to influence the miR-133a level in the established cardiac hypertrophy model. As shown in Fig. 3A, left panel, the expression level of miR-133a was found significantly decreased in TAC-treated mice, but its expression was up-regulated nearly to the normal level by choline and specific inhibition of the $\mathrm{M}_{3} \mathrm{mAChR}$ signaling pathway abrogated this upregulation. Similar results were obtained under in vitro conditions (Fig. 3A, right 
panel). We also detected the expression level of calcineurin, an important target gene of miR-133a [11].The protein level of calcineurin was significantly increased in TAC hypertrophic mice (Fig. 3B, left panel) and ISO-induced hypertrophic cardiomyocytes (Fig. 3B, right panel) as well, while choline significantly suppressed the expression of calcineurin. Moreover, the choline-induced downregulation of calcineurin expression was mitigated by 4-DAMP. Similar results were obtained from cultured NRVCs (Fig. 3B).

\section{Network analysis of potential mechanisms underlying the anti-hypertrophic effects of choline}

In this study, the chemical-gene association database STITCH 3.0 [16] was retrieved for the high-confidant choline-gene associations in human, and the Cytoscape [17] plugins DisGeNET [18] and
BisoGenet [19] were applied to obtain cardiac hypertrophy-related genes and experimental human protein-protein interactions. As the results of our network analysis showed, choline was not found to be functionally associated with any proteins encoded by cardiac hypertrophy-related genes (Fig. 4). However, all its associated proteins including $\mathrm{M}_{3} \mathrm{mAChR}$ and the other four proteins have the potential to directly interact with the cardiac hypertrophy-related proteins. Furthermore, Figure 4 also illustrated that the cardioprotective effects of choline against the TAC-induced hypertrophy could not be exclusively explained by activation of the $\mathrm{M}_{3} \mathrm{mAChR}$ pathway, although it was an indispensable participant; on the other hand, the other four choline-associated proteins might participate in the $\mathrm{M}_{3} \mathrm{mAChR}$ signaling pathways, implying that the implement of their roles still depend on the activation of $\mathrm{M}_{3} \mathrm{mAChR}$.
A

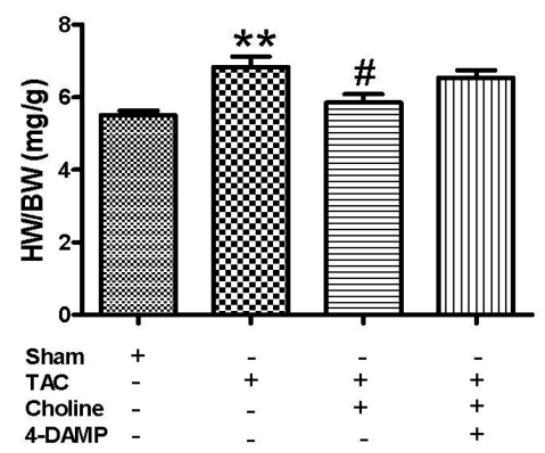

C

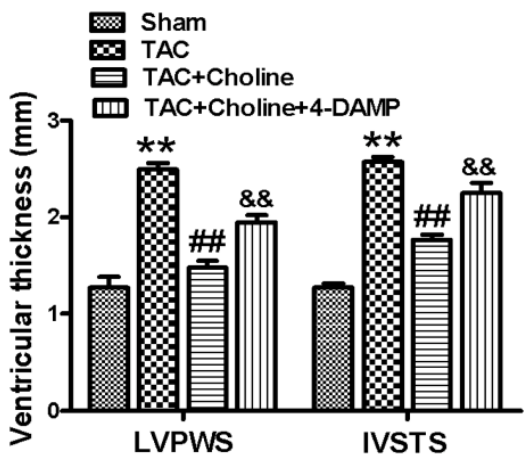

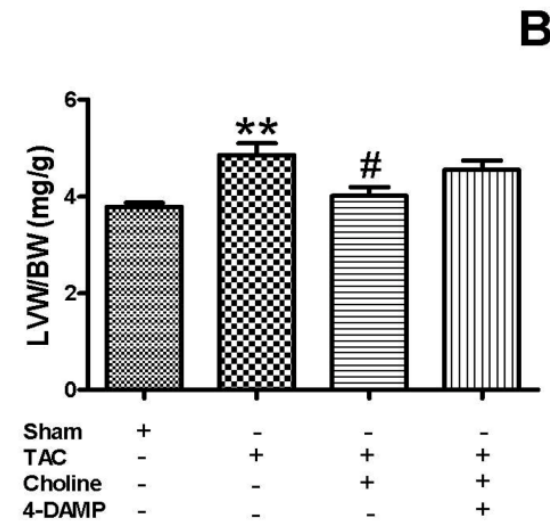

D E

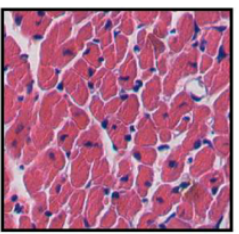

Sham

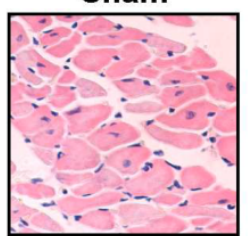

TAC

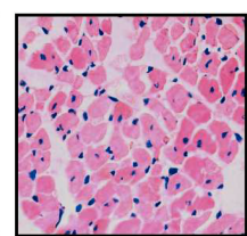

TAC+Choline
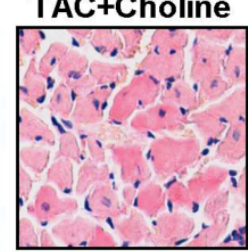

TAC+Choline+4-DAMP
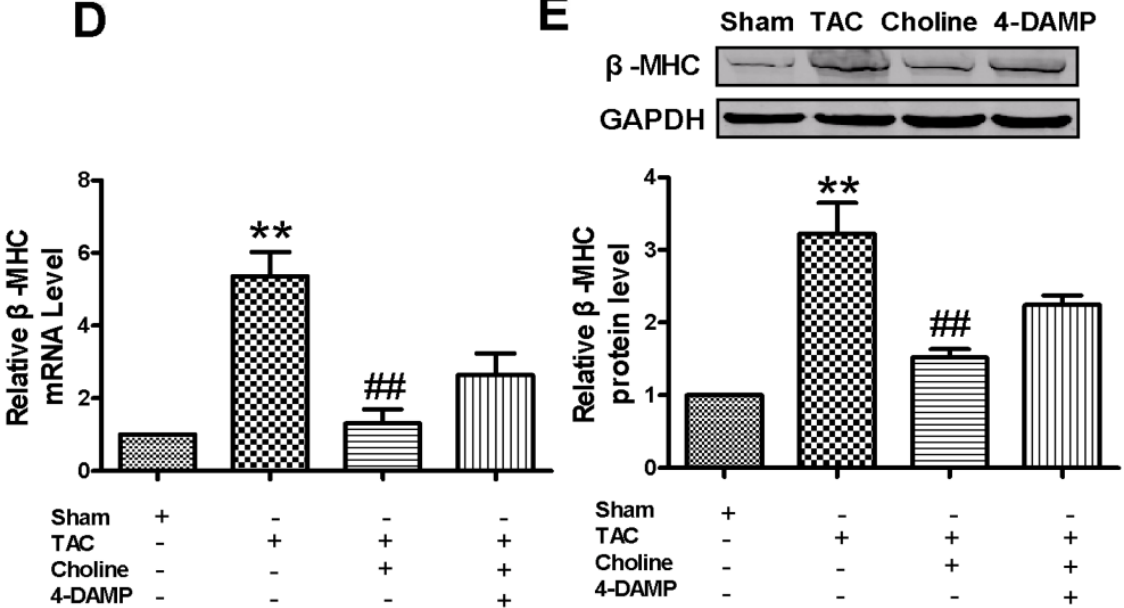

Figure I. Effects of choline on TAC-induced cardiac hypertrophy. (A) Comparison of HW/BW and LVW/BW. Sham $(n=9) ;$ TAC $(n=10) ;$ TAC + Choline $(n=1 I) ;$ TAC + Choline + 4-DAMP $(n=8) .{ }^{* *} p<0.01$ vs sham; ${ }^{*} p<0.05$ vs TAC. (B) Representative H\&E staining of heart trans-section in the four experimental groups. (C) Echocardiographic characteristics of mice in each group. LVPWS: left ventricular posterior systolic wall thickness; IVSTS: interventricular septal systolic thickness; $(n=5),{ }^{* *} p<0.01$ vs Sham; ${ }^{\#} p<0.01$ vs TAC; ${ }^{2 \&} p<0.01$ vs TAC+Choline. (D) Effects of choline on the mRNA expression of $\beta-M H C$ in the hypertrophic left ventricular tissue. ${ }^{* *} p<0.01$ vs Sham; ${ }^{\# \#} p<0.01$ vs TAC. (E) Effects of choline on the protein expression of $\beta-M H C$ in the hypertrophic left ventricular tissue. Data are expressed as mean \pm SEM for 3-4 individual experiments; ${ }^{* *} p<0.01$ vs Sham; ${ }^{\#} p<0.01$ vs TAC. 
A

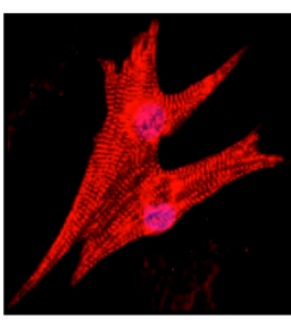

Ctrl

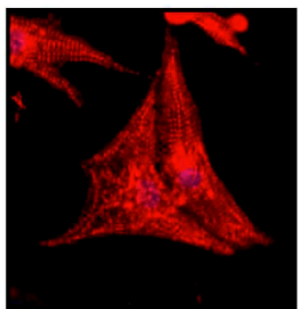

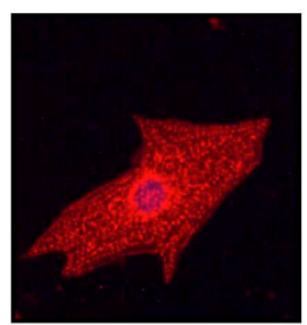

ISO

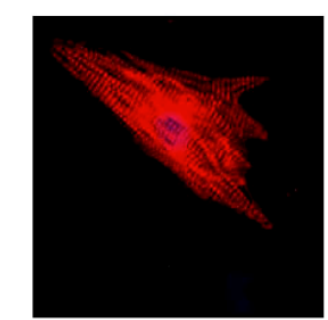

\section{Choline+ISO 4-DAMP+Choline+ISO}

C

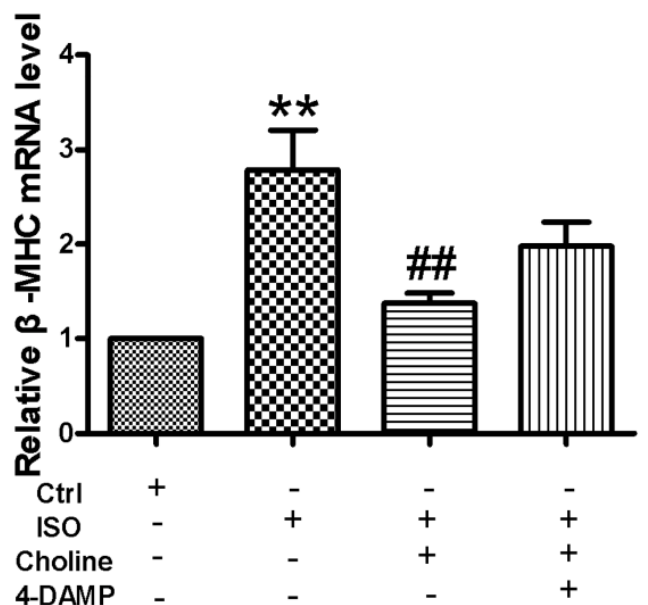

B

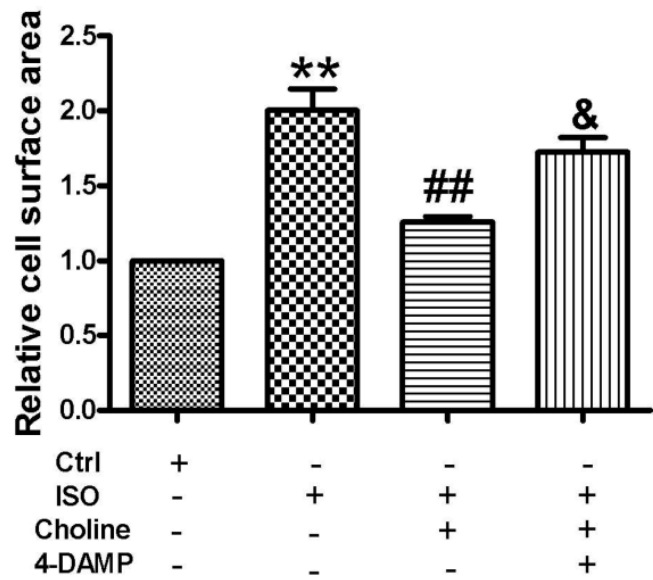

D
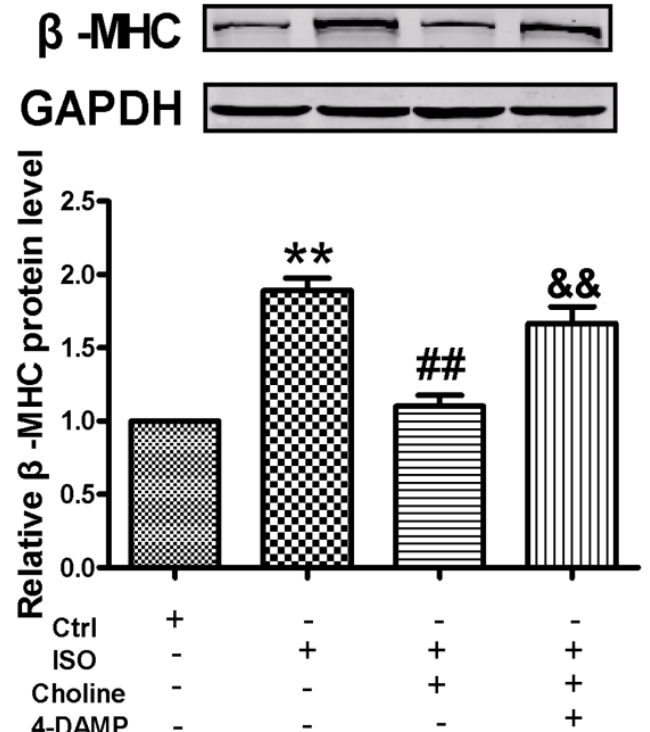

Figure 2. Effects of choline on ISO-induced cardiomyocytes hypertrophy. (A) Representative immunofluorescence staining of cardiomyocytes was observed $(\times 200)$ with $\alpha$-actinin antibody (red signal) and nuclei were stained with DAPI (blue signal). (B) Statistical analysis of cell surface area. Ctrl ( $\mathrm{n}$

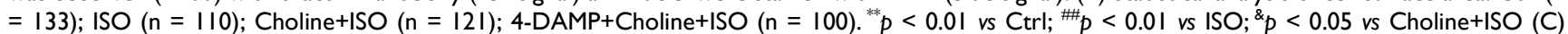
Regulation of the mRNA expression of $\beta$-MHC. ${ }^{* *} p<0.0$ I vs Ctrl; ${ }^{\#} p<0.0$ I vs ISO. (D) Representative western blot of $\beta-M H C$ protein in vitro. Data are expressed as mean \pm SEM for 3-4 individual experiments; $p<0.01$ vs Ctrl; ${ }^{*} p<0.01$ vs ISO; ${ }^{*} p<0.01$ vs $C h o l i n e+I S O$. 
A

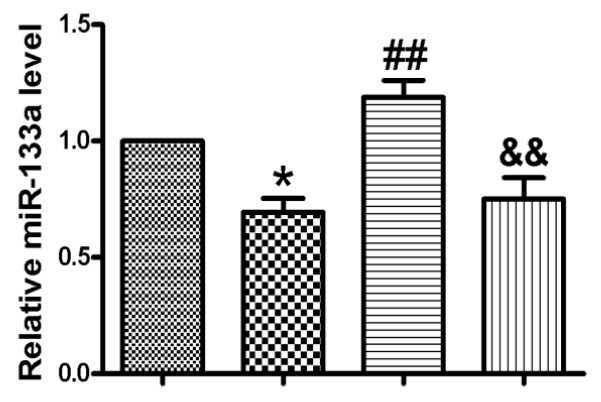

B
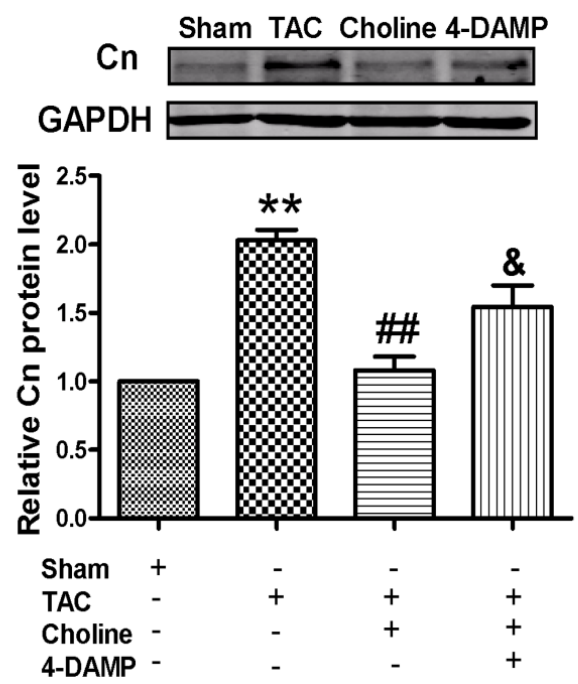

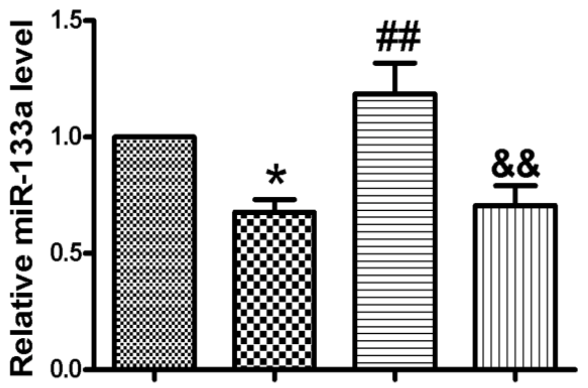

$\begin{array}{ccccc}\text { Ctrl } & + & - & - & - \\ \text { ISO } & - & + & + & + \\ \text { Choline } & - & - & + & + \\ \text { 4-DAMP } & - & - & - & +\end{array}$
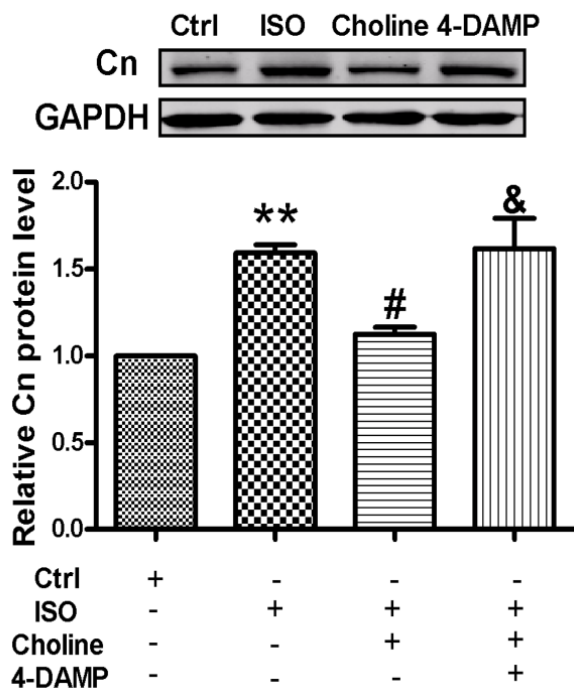

Figure 3. Effects of choline on miR-I33a and calcineurin expression. (A) Expression of miR-I33a was increased by choline in vivo (left panel) and in vitro (right panel). " $p<0.05$ vs Sham-Ctrl; ${ }^{*} p<0.0$ I vs TAC-ISO; ${ }^{2 \&} p<0.05$ vs TAC+Choline - Choline+ISO; (B) Inhibitory effects of choline on the expression of calcineurin in cardiac tissue (left panel) and cardiomyocytes (right panel). Data are expressed as mean \pm SEM for 3-4 individual experiments; ${ }^{* *} p<0.01$ vs Sham-Ctrl; ${ }^{\#} p<0.01$ vs TAC; ${ }^{*}<0.05$ vs ISO; ${ }^{*}<0.05$ vs TAC+Choline -Choline+ISO.

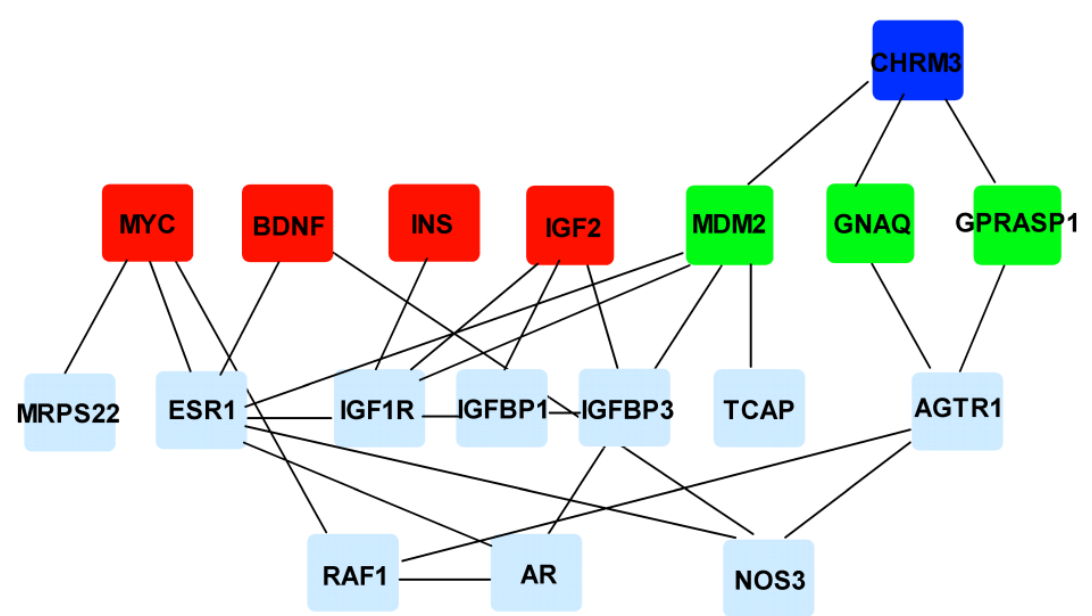

Figure 4. Results of network analysis. Red nodes represent the choline-associated proteins; deep blue and green nodes represent its receptor M3 $\mathrm{mAChR}$ and the downstream proteins of $\mathrm{M} 3 \mathrm{mAChR}$, respectively; light nodes represent the proteins encoded by cardiac hypertrophy related genes; edges represent experimentally validated protein-protein interactions. CHRM3: cholinergic receptor, muscarinic 3; MYC: myelocytomatosis oncogene; BNDF: brain-derived neurotrophic factor; INS: insulin; IGF2: insulin-like growth factor 2; GNAQ: guanine nucleotide binding protein (G protein), q polypeptide; GPRASPI: G protein-coupled receptor associated sorting protein I; MRPS22: mitochondrial ribosomal protein S22; ESR I: estrogen receptor I; IGFIR: insulin-like growth factor I receptor; IGFBPI/3: insulin-like growth factor binding protein I/3; TCAP: titin-cap; AGTRI: angiotensin II receptor, type I; RAFI: v-raf-leukemia viral oncogene I; AR: androgen receptor; NOS3: nitric oxide synthase 3. 


\section{Discussion}

At present, we validated the cardioprotective effects of supplemental choline on TAC-induced cardiac hypertrophy. The TAC pressure-overload and ISO-induced hypertrophic models were employed in this study [20]. Our study generated strong evidence that choline ameliorate cardiac hypertrophy, as indicated by the reduced heart weight, left ventricular weight, ventricular thickness, and reduced expression of biomarker genes of cardiac hypertrophy. As an agonist of $\mathrm{M}_{3} \mathrm{mAChR}$, choline has been shown to produce an important role in ischemic myocardial protection $[4,5,12,13]$, but the possible effects of choline on cardiac hypertrophy was unstudied. Thus, our finding that choline produces anti-hypertrophic effect unraveled a novel aspect of cardiac protection afforded by $\mathrm{M}_{3} \mathrm{mAChR}$. Our network analysis further revealed that MYC, BDNF, INS and IGF2 may be involved in the $\mathrm{M}_{3} \mathrm{mAChR}$ signaling pathways, contributing to the protective effects of choline via their functional associations with this small molecule [21-24]. More functional experiments are needed to elucidate their respective contributions to the effects of choline.

Another important finding in our study was the rescuing of abnormally downregulated expression miR-133a and restoration of calcineurin protein level by choline. The expression level of miR-133a has been demonstrated to be markedly downregulated under hypertrophic conditions of various in vivo and in vitro models [10,25]. Manifold experiments suggest that calcineurin is a key signaling molecule that is upregulated during the development of cardiac hypertrophy $[11,26,27]$ and has been shown to be an important target gene of miR-133a [11]. Normalization of deregulated miR-133a and calcineurin expression has been thought to produce anti-hypertrophic action $[10,11,26,28]$. Interestingly, we found here that miR-133a expression was decreased and calcineurin protein level was increased in the cardiac hypertrophy. The results were in line with the data from intraoperative biopsies from 46 patients with aortic stenosis [29]. Notably, the effects of choline were all abolished by the $\mathrm{M}_{3} \mathrm{mAChR}$-specific antagonist 4-DAMP. These findings suggest that the anti-hypertrophic action of choline is conferred by its ability to activate $\mathrm{M}_{3} \mathrm{mAChR}$ and correct the deregulated expression of miR-133a and calcineurin.

In conclusion, our study unraveled for the first time the cardioprotection of choline against cardiac hypertrophy. Multiple signaling pathways might be involved in it, strongly suggesting complex mechanisms for the anti-hypertrophic effects of choline.
Nevertheless, our findings not only broadened our understanding of the actions of choline in heart disease but also suggested the potential of the essential nutrient choline in the prevention and treatment of cardiac hypertrophy, a main cause of lethal heart failure in clinic.

\section{Supplementary Material}

Figure S1 and Table S1.

http://www.ijbs.com/v09p0295s1.pdf

\section{Acknowledgement}

This work was supported by National Natural Science Foundation of China (Grant No. 81072639).

\section{Conflict of Interests}

The authors have declared that no competing interests exist. Competing Interests

\section{References}

1. Zeisel SH, da Costa KA. Choline: an essential nutrient for public health. Nutr Rev. 2009; 67: 615-623.

2. Homocysteine Studies Collaboration. Homocysteine and risk of ischemic heart disease and stroke: a meta-analysis. JAMA. 2002; 288: 2015-2022.

3. Glunde K, Bhujwalla ZM, Ronen SM. Choline metabolism in malignant transformation. Nat Rev Cancer. 2011; 11: 835-848.

4. Pan Z, Guo Y, Qi H, Fan K, Wang S, Zhao H, Fan Y, Xie J, Guo F, Hou Y, Wang N., Huo R, Zhang Y, Liu Y, Du Z. M3 Subtype of Muscarinic Acetylcholine Receptor Promotes Cardio- protection via the Suppression of miR-376b-5p. PLoS One. 2012; 7:e32571.

5. Yang BF, Lin HX, Xu CQ, Liu Y, Wang H, Han H, Wang Z. Choline produces cytoprotective effects against ischemic myocardial injuries: Evidence for the role of cardiac $\mathrm{M}_{3}$ subtype muscarinic acetylcholine receptors. Cell Physiol Biochem. 2005; 16: 163-174.

6. Liu Y, Sun HL, Li DL, Wang LY, Gao Y, Wang YP, Du ZM, Lu YJ, Yang BF. Choline produces antiarrhythmic actions in animal models by cardiac M3 receptors: improvement of Intracellular $\mathrm{Ca}^{2+}$ handlingas a commonmechanism. Can J Physiol Pharmacol. 2008; 86: 860-865.

7. Wang S, Han HM, Pan ZW, Hang PZ, Sun LH, Jiang YN, Song HX, Du ZM, Liu Y. Choline inhibits angiotensin II-induced cardiac hypertrophy by intracellular calcium signal and p38 MAPK pathway. Naunyn-Schmiedeberg's Arch Pharmacol. 2012; 385: 823-831.

8. Balakumara $P$, Jagadeeshb G. Multifarious molecular signaling cascades of cardiac hypertrophy: Can the muddy waters be cleared? Pharmacological Research. 2010; 62: 365-383.

9. Bernardo BC, Weeks KL, Pretorius L, McMullen JR. Molecular distinction between physiological and pathological cardiac hypertrophy: Experimental findings and therapeutic strategies. Pharmacology \& Therapeutics. 2010; 128: 191-227.

10. Carè A, Catalucci D, Felicetti F, Bonci D, Addario A, Gallo P, Bang ML,et al. MicroRNA-133 controls cardiac hypertrophy. Nature medicine. 2007; 13: 613-618.

11. Dong DL, Chen C, Huo R, Wang N, Li Z, Tu Y, Chu X, Huang W, Yang BF. T Reciprocal repression between microRNA-133 and calcineurin regulates cardiac hypertrophy: a novel mechanism for progressive cardiac hypertrophy. Hypertension. 2010; 55: 946-952.

12. Hang PZ, Zhao JL, Wang YP, Sun LH, Zhang Y, Yang LL, Zhao N, Sun ZD, Mao Y, Du ZM. Reciprocal regulation between M3 muscarinic acetylcholine receptor and protein kinase $\mathrm{C}-\varepsilon$ in ventricular myocytes during myocardial ischemia in rats. Naunyn-Schmied Arch Pharmacol. 2009; 380: 443-450.

13. Wang YP, Hang PZ, Sun $L H$, Zhang $Y$, Zhao JL, Pan ZW, Ji H, Wang L, $\mathrm{Bi} \mathrm{H}, \mathrm{Du} \mathrm{ZM} . \mathrm{M}_{3}$ muscarinic acetylcholine receptor is associated with $\beta$-catenin in myocytes duringmyocardial infraction in the rat. Clinical and Experimental Pharmacology and Physiology. 2009; 36: 995-1001.

14. Zhao J, Su Y, Zhang Y, Pan Z, Yang L, Chen XC, Liu Y, Lu YJ, Du ZM, Yang BF. Activation of cardiac muscarinic $\mathrm{M} 3$ receptors induces delayed cardioprotection by preserving phosphorylated connexin 43 and upreg- 
ulating cyclooxygenase-2 expression. Br J Pharmacol. 2010; 159: 1217-1225.

15. Liao $Y$, Ishikura $F$, Beppu $S$, Asakura $M$, Takashima $S$, Asanuma $H$, Sanada S, Kim J, Ogita H, Kuzuya T, Node K, Kitakaze M, Hori M. Echocardiographic assessment of LV hypertrophy an function in aortic-banded mice: necropsy validation. Am Physiol Heart Circ Physiol. 2002; 282: H1703-H1708.

16. Kuhn M, Szklarczyk D, Franceschini A, Csmpillos M, Von M, Jensen LJ, Beyer A, Bork P. STITCH 2: an interaction network database for small molecules and proteins. Nucleic Acids Res. 2010; 38(Database issue): D552-D556.

17. Shannon P, Markiel A, Ozier O, Baliga NS, Wang JT, Ramage D, Amin N, Schwikowski B, Ideker T. Cytoscape: a software environment for integrated models of biomolecular interaction networks. Genome Res 2003;13: 2498-2504.

18. Bauer-Mehren A, Rautschka M, Sanz F, Furlonget L. DisGeNET: a Cytoscape plugin to visualize, integrate, search and analyze gene-disease networks. Bioinformatics. 2010; 26: 2924-2926.

19. Martin A, Ochagavia ME, Rabasa LC, Miranda J, Fernandez-de-Cossio J. BisoGenet: a new tool for gene network building, visualization and analysis. BMC Bioinformatics 2010; 11: 91.

20. Berry JM, Naseem RH, Rothermel BA, Hill J. Models of cardiac hypertrophy and transition to heart failure. Drug Discovery Today: Disease Models. 2007; 4: 198-206.

21. Sesca E, Chiara M, Binasco V, Tessitore L. The delay in rat liver regeneration by choline is associated to alteration in c-myc expression. Boll Soc Ital Biol Sper. 1996; 72: 217-222.

22. Johansson J, Formaggio E, Fumagalli G, Chiamulera C. Choline up-regulates BDNF and down-regulates TrkB neurotrophin receptor in rat cortical cell culture. Neuroreport. 2009; 20: 828-832.

23. Cazzolli R, Huang P, Teng S, Hughes WE. Measuring phospholipase D activity in insulin-secreting pancreatic beta-cells and insulin-responsive muscle cells and adipocytes. Methods Mol Biol. 2009; 462: 241-251.

24. Napoli I, Blusztajn JK, Mellott TJ. Prenatal choline supplementation in rats increases the expression of IGF2 and its receptor IGF2R and enhances IGF2-induced acetylcholine release in hippocampus and frontal cortex. Brain Res. 2008; 1237: 124-135.

25. Li $Q$, Lin $X$, Yang $X$, Chang J. NFATc4 is negatively regulated in miR-133a-mediated cardiomyocyte hypertrophic repression. Am J Physiol Heart Circ Physiol. 2010; 298: H1340- 1347.

26. Tan XY, Li JP, Wang XY, Chen N, Cai BZ, Wang G, Shan HL, Dong DL, Liu YJ, Li XD, Yang F, Li X, Zhang P, Li XQ, Yang BF, Lu YJ. Tanshinone IIA Protects Against Cardiac Hypertrophy via Inhibiting Calcineurin/Nfatc3 Pathway. Int J Biol Sci. 2011; 7: 383-389.

27. Chen C, Huo R, Tong Y, Sheng Y, Liu HB, Gao X, Nakajima O, Yang BF, Dong DL. Systemic Heme Oxygenase-1 Transgenic Overexpression Aggravates Pressure Overload-induced Cardiac Hypertrophy in Mice. Cell Physiol Biochem. 2011; 28: 25-32.

28. Donaldson C, Eder S, Baker C, Aronovitz MJ, Weiss AD, Hall-Porter M, Wang F, Ackerman A, Karas RH, Molkentin JD, Patten RD. Estrogen attenuates left ventricular and cardiomyocyte hypertrophy by an estrogen receptor-dependent pathway that increases calcineurin degradation. Circ Res. 2009; 104: 265-275.

29. Villar AV, Merino D, Wenner M, Liano M, Cobo M. Myocardial gene expression of microRNA-133a and myosin heavy and light chains, in conjunction with clinical parameters, predict regression of left ventricular hypertrophy after valve replacement in patients with aortic stenosis. Heart. 2011; 97: 1132-1137. 